\title{
Epistemología y su vigencia en el quehacer investigativo universitario
}

\section{Epistemology and its validity in university research}

\author{
María Rosario Álava Zambrano \\ rosarioalava85@gmail.com \\ Universidad Laica Eloy Alfaro de Manabí, Manta \\ Ecuador \\ https://orcid.org/0000-0002-5223-7566 \\ Pedro Vicente Quijije Moreira \\ gpmpv.1962@hotmail.com \\ Universidad Laica Eloy Alfaro de Manabí, Manta \\ Ecuador \\ https://orcid.org/0000-0002-4221-3704 \\ José Rafael Álava Zambrano \\ rafa89.raz@gmail.com \\ Universidad Laica Eloy Alfaro de Manabí, Manta \\ Ecuador \\ https://orcid.org/0000-0002-2228-9998
}

Recibido: 15 de agosto de 2019

Aprobado: 14 de septiembre de 2019

\section{RESUMEN}

El artículo de revisión tiene por esencia un análisis sobre la epistemología y su vigencia en el quehacer investigativo universitario, se conciben diversas concepciones que permiten el abordaje de la investigación en las universidades, posibilitando el encuentro del investigador con el objeto - sujeto - fenómeno investigado, en función del abordaje epistemológico - metodológico, el objeto concuerda con la concepción heredada, el sujeto con la reflexión libre, el fenómeno desde la introspección de Ricci, así se condicionan los procesos investigativos para configurar un accionar concomitante de reflexión y de acción por parte del investigador. La epistemología se encuentra vigente como estructura coherente de los procesos investigativos en las universidades, sin embargo, se enfrenta al reto de redescubrir la esencia de lo que es investigar, porqué y paraqué se investiga en las universidades, cuales son las finalidades 
últimas que permiten ejecutar investigaciones con pertinencia al crecimiento integral de la sociedad.

Descriptores: Epistemología; Estructura del conocimiento; Creencia; investigación.

\begin{abstract}
The review article is essentially an analysis of the epistemology and its validity in the university research work, various conceptions are conceived that allow the approach of research in universities, making it possible for the researcher to meet the object - subject - phenomenon investigated, depending on the epistemological - methodological approach, the object agrees with the inherited conception, the subject with free reflection, the phenomenon from Ricci's introspection, thus the investigative processes are conditioned to configure a concomitant action of reflection and action by the investigator. Epistemology is in force as a coherent structure of research processes in universities, however, it faces the challenge of rediscovering the essence of what it is to investigate, why and why research in universities, which are the ultimate purposes that allow executing research relevant to the integral growth of society.
\end{abstract}

Descriptors: Epistemology; Structure of knowledge; Belief; Research.

\title{
DESARROLLO
}

La epistemología en su concepción genérica se considera como la ciencia del conocimiento o como aquella que busca estudiar la validez del conocimiento, en la actual investigación, no se procura estudiar la esencia del concepto, se aborda su vigencia en el quehacer investigativo universitario, por cuanto la epistemología ciertamente se encuentra relacionada directamente con la investigación científica y académica. Así el docente universitario en su responsabilidad o rol de ser investigador, debe asumir la epistemología en conjunto con la metodología como dos aristas necesarias para realizar investigaciones coherentes desde el punto de vista del conocimiento a generar y como generarlo.

En ese sentido, la epistemología puede ser generada desde diversas concepciones del pensamiento filosófico, entre los cuales se encuentran la concepción heredada del 
Círculo de Viena, perspectiva de reflexión libre, meta- teoría (Padrón, 2007). Así mismo Jaramillo (2003), concibe la epistemología como aquella que "se escenifica en la interacción, por cuanto no se anida en la estructura de un pensamiento interno solipsístico, sino también en la interacción que se tiene con el otro; es decir, es una epistemología producto de una reflexión compartida" (p. 178). Por otro lado, Ricci (1999), comenta:

Epistemología es pues, a mi criterio, ese punto de vista desde el cual me relaciono con las cosas, con los fenómenos, con los hombres y eventualmente con lo trascendente. Esto, que se produce en el ámbito personal y cotidiano, también ocurre en el ámbito científico, donde proliferan distintas corrientes y sistemas de pensamiento que resultan ser, en definitiva, formas de ver el mundo. Estas epistemologías "verán" el contexto de producción del conocimiento así como el contexto de su validación y aplicación desde su punto de vista, es decir desde donde ellas encuentran su sustentación crítica (p. 2).

Lo abordado hasta ahora, permite evaluar tres concepciones sobre epistemología, la visión de Padrón, permite clasificar la epistemología en tres bloques o contextos desde el cual se puede hacer investigación de forma secuencial diferente, es decir, la concepción heredada parte del positivismo lógico y se complementa con el falsacionismo popperiano y desde el socio historicismo kuhniano. La concepción de reflexión libre se construye sobre la base de nociones tales como el "pensamiento complejo", el "holismo", la "transdisciplinariedad", la "reflexividad" o la "posmodernidad". Mientras que la visión meta- teoría, se encuentra obligada a explicar, mediante teorías contrastables, los procesos del conocimiento científico.

En el caso de Jaramillo, la epistemología se sostiene y aporta en la medida que se genera conocimiento desde la interacción interpersonal, lo cual por medio de la intersubjetividad genera conocimientos en donde lo científico - cotidiano se complementa para brindarle significados al objeto o fenómeno de investigación, posibilitando al investigador el análisis de contenido del mensaje, con la finalidad de construir conocimientos. 
La perspectiva de Ricci, además de lo intersubjetivo, tiene en cuenta la introspección, proceso que permite al sujeto generar conocimientos desde su propia reflexión o praxis, aunado de tener en cuenta lo científico - cotidiano. Así el investigador puede generar conocimientos científicos desde una perspectiva mixta de lo metodológico, integrando técnicas que posibilitan la decodificación de los significados otorgados a los datos, Colina \& Aldana (2017), señalan que "la formación a lo largo de la vida representa procesos de internalización, externalización, socialización, combinación, para la creación de nuevos conocimientos, teniendo como eje importante de la dimensión humana" ( $p$. 38).

Se conciben diversas concepciones epistemológicas que permiten el abordaje de la investigación en las universidades, posibilitando el encuentro del investigador con el objeto - sujeto - fenómeno investigado, en función del abordaje epistemológico metodológico, el objeto concuerda con la concepción heredada, el sujeto con la reflexión libre y la postura de Jaramillo, el fenómeno desde la introspección de Ricci, así se condicionan los procesos investigativos para configurar un accionar concomitante de reflexión y de acción por parte del investigador.

En ese sentido, Aldana \& Colina (2017), plantean que desde la reflexión - acción, se abre "paso a la planificación centrada en la investigación, develando la reducción a su mínima expresión de la investigación por medio de los esquemas institucionalizados en la investigación; ofreciendo una alternativa como es La investigación para la transformación social” (p. 9). La visión epistemológica de la transformación social parte desde la concepción intersubjetiva, pero no como mera interpretación de significados, sino, como acción transformadora de la realidad en donde se conforman equipos de investigación bajo una figura cooperativa de interacción sinérgica para la generación de soluciones y luego de conocimientos, siendo una episteme para la gestión investigativa social. 
Desde la visión de la acción transformadora, se pueden integrar la introspección además de la intersubjetividad y reflexión libre, como procesos complementarios que permiten fusionar visiones epistémicas con la finalidad de equiparar la investigación a la concepción global de mundo donde se transita actualmente, así, la investigación universitaria cobra vigencia, por ende la epistemología como ciencia en procura de generar un proceso coherente para la estructuración de una investigación en esencia de los intereses del investigador.

En complemento de lo expuesto, nace la visión del investigador social universitario, teniendo como esencia "convertirse en un líder transformador, comprometido con su entorno social, en donde su palabra tenga poder reflexivo, crítico y emancipador, en la construcción y reconocimiento de nuevos saberes" (Aldana, 2017, p. 101). Desde lo abordado, el investigador universitario se redescubre para tributar entre la lógica teórica de las aulas hacia el accionar cotidiano del contexto social, así la universidad deja de ser una representación social de instrucción, para constituirse en un agente de construcción de múltiples realidades a la luz de concebir la investigación como proceso central de su accionar.

La epistemología desde las nuevas concepciones del pensamiento y de necesidades de acción de la sociedad global, regenera su accionar, permitiendo estudiar diversas concepciones específicas de los grupos sociales, una de ellas por ejemplo, la perspectiva de género, aunque Guzmán \& Pérez (2005), advierten de la debilidad teórica de esta visión, sin embargo, como tendencia social posmoderna, será necesario estudiar su evolución en próximas investigaciones con la finalidad de percibir su aporte al mundo de la investigación científica en coherencia epistemológica. Aldana Zavala (2019), considera que:

La epistemología en el contexto universitario latinoamericano transita hacia la consecución de unas ciencias sociales que, mediante la investigación, pueda germinar conocimientos científicos en contexto social, con la finalidad de brindar la orientación hacia una identidad investigativa fundamentada desde la epistemología misma. Para esto es pertinente la formación continua sobre filosofía y epistemología en las universidades, pues solo así se podrá 
conocer, reconocer, valorar, pero sobre todo transcender hacia nuevos estados de la investigación universitaria en Latinoamérica, donde se tenga en cuenta la investigación como el factor primordial para el progreso de la sociedad (p. 9).

La formación en filosofía y por ende en epistemología es una urgente necesidad en las universidades, no solo para los docentes investigadores, sino, para la comunidad estudiantil, por cuanto esto permite generar una postura crítica sobre la realidad social, abordada desde la investigación universitaria, esto permitirá además transcender escenarios donde solo se ejecutan trabajos de investigación por el solo hecho de aprobar una determinada evaluación, hacia una donde se realice investigación en conformidad de promover aportes significativos en la consolidación de las políticas públicas de los planes de desarrollo nacional y regional.

La epistemología e investigación universitaria, cobran motivación desde la necesaria concatenación en contribuir en la conexión de lo político - social, los países latinoamericanos no pueden estar desconectados con la investigación universitaria, desde adoptarse el enfoque de bienestar social y no del asistencialismo investigativo, consistiendo este último, en meros financiamientos de proyectos que en ocasiones no tributan a contribuciones eficaces desde la universidad hacia lo social.

Rodríguez (2019), comenta la urgente necesidad de un paradigma emergente que posicione la universidad como motor investigativo desde investigaciones pertinentes al crecimiento integral de la sociedad. Borrero (2019), en complemento plantea una gestión educativa enfocada en fusionar lo institucional y comunitario como un bloque para la germinación de soluciones tangibles a la sociedad. lanni Gómez (2017), advierte que "para desarrollar aprendizajes desde la investigación, la introspección reflexiva, la crítica, como elementos que transversalicen toda la formación se requiere de un modelo educativo consustanciado y perfilado desde estos componentes dinamizadores" (p. 15). El redescubrir la vigencia de la epistemología, trae consigo el develar la necesidad de un currículo dinámico, flexible, Castro\& Isea Argüelles (2019), enfatizan que además este debe contemplar la coexistencia de la universidad y comunidad, mediante una acción 
social transformadora y no asistencialista. Alemán Sangronis (2019), comenta la pertinencia de descolonizar el quehacer investigativo con la finalidad de reconstruir su perspectiva de aporte significativo a la sociedad, mientras que Rodríguez Acasio (2018), expone la urgente de una nueva gerencia educativa enfocada desde la investigación con el propósito de construir un paradigma gerencial sustentado desde la investigación universitaria, en complemento, Rodríguez Acasio, \& Colina Ysea (2016), destacan:

La función investigación y su gestión, presenta una cultura investigativa muy débil; las estructuras que existe son rígidas, cerradas y obsoleta, ya que se priva la libertad del pensamiento del investigador; la gestión administrativa es lenta, pasiva y burocrática, por lo que no responde a las necesidades de sus investigadores, considerando que son sumamente procedimentales, legalistas y cumplidores de normas (p. 12).

Se observa la existencia de un problema de fondo, el cual epistemológicamente debe ser develado para confrontar la realidad con lo que se pretende lograr realmente en las universidades desde la praxis investigativa, por lo tanto, no se puede condicionar a simples programas de capacitación sobre investigación, es necesario como se mencionó en líneas anteriores, formar en filosofía y epistemología como un estilo de formación profesional permanente, esto brindará progresivamente el espacio auto y co reflexivo de los investigadores universitarios con la finalidad de abordar múltiples realidades en contexto de transformar el paradigma de reproducción al de transformación investigativa en contexto con las perspectivas de la sociedad global.

\section{REFLEXIONES DE CIERRE}

La epistemología se encuentra vigente como estructura coherente de los procesos investigativos en las universidades, sin embargo, se enfrenta al reto de redescubrir la esencia de lo que es investigar, porqué y paraqué se investiga en las universidades, cuales son las finalidades últimas que permiten ejecutar investigaciones con pertinencia al crecimiento integral de la sociedad, aunado que al tener la perspectiva de la investigación como centro del aprendizaje, se trastoca las bases del currículo universitario, contemplando la urgente necesidad de promover su actualización en virtud 
de los nuevos desafíos globales, investigar y educar se complementan, a la vez que se fusionan en una visión que debe ser promovida como el real desafío epistemológico, por cuanto el investigador social universitario no internalice el valor de la epistemología, difícilmente adoptará nuevas perspectivas educativas e investigativas.

\section{REFERENCIAS CONSULTADAS}

1. Aldana Zavala, J. J. (2019). La competencia epistemológica en el investigador social universitario venezolano. Praxis, 15(1). https://doi.org/10.21676/23897856.3091

2. Aldana Zavala, J.J. (2017). La investigación social. Competencias en el docente universitario. ISBN: 978-980-7792-3-4. Recuperado de http://fundacionkoinonia.com.ve/publicaciones/Competencias Investigativas2.pdf

3. Aldana \& Colina (2017). La visión paradigmática de la investigación en el contexto universitario. Ciencias Sociales y Educativa UNEFM, 7(1), 9 - 15. Recuperado de https://rcseunefm.files.wordpress.com/2017/09/rcsevolviinro1.pdf

4. Alemán Sangronis, J. (2019). Descolonización del hacer investigativo para la Regeneración Social Venezolana. CIENCIAMATRIA, 5(9), 187 - 198. https://doi.org/10.35381/cm.v5i9.112

5. Borrero, R. (2019). Procesos de gestión del talento humano en el sector educativo gerencial de Colombia. Revista Arbitrada Interdisciplinaria Koinonía, 4(7), 293-307. doi:http://dx.doi.org/10.35381/r.k.v4i7.206

6. Castro, M., \& Isea Argüelles, J. (2019). Responsabilidad Social para la Interacción Comunitaria en contextos universitarios: Una aproximación teórica. CIENCIAMATRIA, 5(9), 168 - 186. https://doi.org/10.35381/cm.v5i9.111

7. Colina, F. \& Aldana, J. (2017). El símbolo: bases epistémicas en la investigación científica. Una lectura crítico - reflexiva. Ciencias Sociales y Educativa UNEFM, 7(2), $\quad 38 \quad-\quad$ 43. $\quad$ Recuperado de https://rcseunefm.files.wordpress.com/2017/09/rcsevolviinro21.pdf

8. Guzmán, M., \& Pérez, A. (2005). Las Epistemologías Feministas y la Teoría de Género. Cinta de Moebio. Revista de Epistemología de Ciencias Sociales, (22). Consultado de https://cintademoebio.uchile.cl/index.php/CDM/article/view/26089/27394 
9. Ianni Gómez., L. (2017). MIRAMDA: Una propuesta educativa emergente desde la investigación. Revista Arbitrada Interdisciplinaria Koinonía, 2(3), 9-30. Recuperado http://fundacionkoinonia.com.ve/ojs/index.php/revistakoinonia/article/view/49/36

10. Jaramillo, L. (2003). ¿Qué es Epistemología? Cinta de Moebio. Revista de Epistemología de Ciencias Sociales, (18). Consultado de https://cintademoebio.uchile.cl/index.php/CDM/article/view/26135/27433

11. Padrón, J. 2007. Tendencias epistemológicas de la investigación científica en el Siglo XXI. Cinta moebio 28: 1-28. Recuperado de https://www.moebio.uchile.cl/28/padron.html

12. Ricci, R. (1999). Acerca de una Epistemología Integradora. Cinta de Moebio. Revista de Epistemología de Ciencias Sociales, (5). Consultado de https://cintademoebio.uchile.cl/index.php/CDM/article/view/26437/27730

13. Rodríguez, A. (2019). Desde la filosofía hacia el pensamiento emergente en el desarrollo de los procesos investigativos. Revista Arbitrada Interdisciplinaria Koinonía, 4(7), 262-279. doi:http://dx.doi.org/10.35381/r.k.v4i7.204

14. Rodríguez Acasio, F. (2018). Gestión de la investigación: Un nuevo paradigma gerencial, desde las Universidades Experimentales. CIENCIAMATRIA, 4(6), 3755. Recuperado a partir de http://cienciamatriarevista.org.ve/index.php/cm/article/view/71

\section{REFERENCES CONSULTED}

1. Aldana Zavala, J. J. (2019). Epistemological competence in the Venezuelan university social
https://doi.org/10.21676/23897856.3091

Praxis, $\quad 15$

(1).

2. Aldana Zavala, J.J. (2017). Social research Skills in the university teacher. ISBN: 978-980-7792-3-4. Recovered http://fundacionkoinonia.com.ve/publicaciones/Competencias Investigativas2.pdf

3. Aldana \& Hill (2017). The paradigmatic vision of research in the university context. Social and Educational Sciences UNEFM, 7 (1), 9 - 15. Recovered from https://rcseunefm.files.wordpress.com/2017/09/rcsevolviinro1.pdf 
4. German Sangronis, J. (2019). Decolonization of investigative activities for the Venezuelan Social Regeneration. SCIENCE, 5 (9), 187-198. Https://doi.org/10.35381/cm.v5i9.112

5. Borrero, R. (2019). Human talent management processes in the management education sector of Colombia. Interdisciplinary Arbitrated Review Koinonía, 4 (7), 293-307. doi: http: //dx.doi.org/10.35381/r.k.v4i7.206

6. Castro, M., \& Isea Argüelles, J. (2019). Social Responsibility for Community Interaction in university contexts: A theoretical approach. CIENCIAMATRIA, 5 (9), 168-186. Https://doi.org/10.35381/cm.v5i9.111

7. Colina, F. \& Aldana, J. (2017). The symbol: epistemic bases in scientific research. A critical - reflexive reading. Social and Educational Sciences UNEFM, 7 (2), 38 $43 . \quad$ Retrieved https://rcseunefm.files.wordpress.com/2017/09/rcsevolviinro21.pdf

8. Guzmán, M., \& Pérez, A. (2005). Feminist Epistemologies and Gender Theory. Moebio tape. Journal of Epistemology of Social Sciences, (22). Retrieved from https://cintademoebio.uchile.cl/index.php/CDM/article/view/26089/27394

9. Ianni Gómez., L. (2017). MIRAMDA: An educational proposal emerging from research. Interdisciplinary Arbitrated Review Koinonía, 2 (3), 9-30. Recovered from http://fundacionkoinonia.com.ve/ojs/index.php/revistakoinonia/article/view/49/36

10. Jaramillo, L. (2003). What is epistemology? Moebio tape. Journal of Epistemology of Social Sciences, (18). Retrieved from https://cintademoebio.uchile.cl/index.php/CDM/article/view/26135/27433

11. Padrón, J. 2007. Epistemological trends of scientific research in the 21st century. Moebio tape 28: 1-28. Recovered from https://www.moebio.uchile.cl/28/padron.html

12. Ricci, R. (1999). About an Integrative Epistemology. Moebio tape. Journal of Epistemology of Social Sciences, (5). Retrieved from https://cintademoebio.uchile.cl/index.php/CDM/article/view/26437/27730

13. Rodríguez, A. (2019). From philosophy to emerging thinking in the development of research processes. Interdisciplinary Arbitrated Review Koinonía, 4 (7), 262-279. doi: http: //dx.doi.org/10.35381/r.k.v4i7.204 


\section{CIENCIAMATRIA}

Revista Interdisciplinaria de Humanidades, Educación, Ciencia y Tecnología

Año VI. Vol. VI. No10. Enero - Julio 2020

Hecho el depósito de ley: pp201602FA4721

ISSN-L: 2542-3029; ISSN: 2610-802X

Universidad Nacional Experimental Francisco de Miranda (UNEFM). Santa Ana de Coro. Venezuela

María Rosario Álava Zambrano; Pedro Vicente Quijije Moreira; José Rafael Álava Zambrano

14. Rodríguez Acasio, F. (2018). Research management: A new managerial paradigm, from the Experimental Universities. SCIENCE, 4 (6), 37-55. Recovered from http://cienciamatriarevista.org.ve/index.php/cm/article/view/71

(C2020 por el autor. Este artículo es de acceso abierto y distribuido según los términos y condiciones de la licencia Creative Commons Atribución-NoComercial-Compartirlgual 4.0 Internacional (CC BY-NC-SA 4.0) (https://creativecommons.org/licenses/by-nc-sa/4.0/). 